\title{
HISTORY OF MATHEMATICS
}

\author{
J. MACCULLAGH AND W.R. HAMILTON \\ THE TRIUMPH OF IRISH MATHEMATICS 1827-1865 \\ N.D. McMillan
}

\section{INTRODUCTION}

In the first article in this series [Newsletter No. 10, pp. 61-75] the reform of the mathematical component of the Dublin University curriculum in the period 1790 to 1831 was described. Ireland took the lead in this period internationally in that Trinity was the first university to introduce into its curriculum the new mathematical methods developed in revolutionary France. The Irish reform. which culminated in the election of Bartholomew Lloyd to the Provostship of Dublin University in 1831 preceded the British reform movement in mathematics.

The University produced two outstanding mathematicians in this second period, James MacCullagh and William Rowan Hamilton. The former established a powerful geometrical research tradition by introducing a new mathematical methodology and by his inspirational teaching methods. Hamilton on the other hand introduced some of the most original and innovative mathematical concepts in the history of the science of algebra. The development of their respective contributions are described chronologically.

The objective of this article is to reveal the nature of the two mathematical methodologies in this Dublin Mathematical School, because it was from the synthesis of these two methodologies that G.F. Fitzgerald was able to found the science of Relativity. 
James Maccullagh and the geOmetric tradition: the quest for the CARTESIAN SYNTHESIS

The geometric reform of the Dublin University curriculum [1] may be dated from 1758 when Euclid was first introduced into the curriculum, but the research tradition was established by James Maccullagh [2]. MacCullagh's geometric ability was unrivalled in his day. His publications are marked by their lucid economic style and their supreme elegance. It is known that he was very conscious of the educational effect of his writings on the minds of his contemporaries, especially his students in Trinity.

Maccullagh's first major paper, on the Rectification of the Conic Sections, was read at the Royal Irish Academy on $21 \mathrm{st}$ June 1830. It was a critique of the mathematical methods used by fresnel in his theoretical studies on the laws of double refraction of light in crystals. MacCullagh presented a series of Lonic theorems aimed at providing the mathematical tools which would enable the theory of light to be placed on a firm geometrical base.

On the 28th May 1832 at the Royal Irish Academy he read a paper on The Theory of Attractions in which he opened up another research interest. In this paper Maccullagh resolved a long-standing dispute over the correctness of Laplace's theory of attraction which had been at issue for a number of years between three of the greatest mathematicians of the period: Laplace, Lagrange and Si. James Ivory. His geometric approach resolved this dispute in favour of Laplace.

After three further years of struggle with his geometrical conceptions, Maccullagh read on the 24 th June 1835 another paper: Geometrical Propositions Applied to the Wave Theory of Light. In this he explained internal and external conical refraction using geometrical principles to replace fresnel's three principles of conservation of vis viva (energy), the uniformity of elasticity of the ether and the continuity of 
displacement parallel to the refracting interface.

On the 9 th January 1837 he produced On the Crystalline Reflexion and Refraction in which he proposed a theory of "great geometric simplicity which was compatible with all previous physical notions" [2]. He predicted results from his theory and compared the results with experimentally known data. The continuity condition was replaced by a principle of equivalent vibrations, which supposed that vibrations in two media are equivalent and these considerations were extended to both the parallel and perpendicular components.

MacCullagh's mathematical description of light propagation was quite remarkable. Despite the difference of mathematical representation today, it is clear that the Maxwellian Equations of Electromagnetism are only MacCullagh's equations with the addition of a single term, the famous displacement curcent [3].

In MacCullagh's next paper, The b:namical Theory of Crystalline Reflexion and Refraction, read at the Academy on the gth December 1839 he deduced all his previous geometrical results from "a single physical hypothesis and from strictly mechanical principles". The full extent of this dynamical theory was presented in The oynamical Theory of light published posthumously by two of his devoted followers [4]. In this theory can be seen the modern mathematical description of light and in fact the equations of electromagnetic fropagation are compatible with MacCullagh's equations.

The contemporaries of Maccullagti accepted a generally held view that his work had established a consistent theory of light. His Royal Irish Academy obituary claimed for him a position of eminence above Fresnel, because it was believed that he had established

"the general equations of the motion of the propagation of light, not only in all known media, but also for all 
media which could ever be discovered, or even conceived."

His honours were of the highest order. The Royal Society in 1842 awarded him the Copley medal, but the recognition by the Royal Irish Academy in awarding him the Cunningham Medal in 1837 was his most treasured honour because of his Irish nationalism.

\section{WILLIAM ROWAN HAMILTON}

A revival of interest in Hamilton can be seen from two recent biographical studies [5, 5a] both of which emphasize his isolated $l i f e$ at Dunsink Dbservatory and his idiosyncrasies. Hamilton was a child prodigy and in particular was noted for his early ability to calculate. He was essentially self-taught in mathematics and probably therefore benefited more from the atmosphere of Trinity under Bartholomew Lloyd than from the actual teaching, since by the time he came to college he was already an accomplished mathematician.

He was by nature an algebraist and like all truly great mathematicians his work revolved around one great idea, in Hamilton's case the Characteristic Function V, which he claimed was the most complete and simple definition that could be given of the application of analysis to optics. This function for him contains the whole of mathematical optics and in this he reveals himself clearly as a follower of Lagrange and Laplace. Hamilton's early work on Caustics [6] contained the germ of the idea, but this idea was first properly exploited in his classic series of memoirs Theory of Systems of Rays. These memoirs appeared between 1827 and 1833 and developed optics merely as an aspect of the calculus of variation. Fermat's idea of least time and Mapertuis' least action principles are in effect exploited by considering all possible curves between two points $A^{\prime}\left(x^{\prime}, y^{\prime}, z^{\prime}\right)$ and $A(x, y, z)$ and selecting a curve giving the smallest value of the integral of the type 


$$
v=\int\left(x, y, z \frac{d x}{d u}, \frac{d y}{d u}, \frac{d z}{d u}\right) d u
$$

The stationary value of this curve is called the rayin optics but is referred to as the extremal in the calculus of variation. The theory is both mathematically and physically notewortriy because it was independent of the physical hypothesis about the nature of light. The wavefronts of the Huygen theory are derived from the direction cosines $\alpha, \beta, \gamma$ of the ray, where these are the partial differential coefficients of the principal function $V$, so that

$$
\alpha=\frac{\partial V}{\partial x}, \quad \beta=\frac{\partial V}{\partial y} \text { and } \gamma=\frac{\partial V}{\partial z} \text {. }
$$

The differential form

$$
\alpha d x+\beta d y+\gamma d z
$$

has to be derived to determine the wavefront, and in optics the solution is simply then obtained by making $V$ the length of the ray.

This method was pregnant with possibilities. Hamilton showed its rower initially when he applied it to a special crystal problem and predicted that in place of a double refraction of light the ray would be refracted into a cone. This startling prediction was made in his classic series of memoirs in 1832 and experimentally confirmed by Humphrey Lloyd [7].

In the development of the properties of extrunals, he made significant discoveries and Synge [8] has pointed out that because of the extreme difficulty of this work it was ignored only to be rediscovered by later mathematicians: Kummer in 1860 on the general theory of rectilinear congruences; Bruns in 1895 rediscovered and renamed the third characteristic function as the image function; and Jacobi developed the theory of infinitesimal contact transformations using only one of Hamilton's equations now known as the Hamilton-Jacobi 
equation.

The next step in Hamilton's mathematical odyssey was to extend his calculus to dynamics. He began this work in 1833 with a paper On the General Method of Expressing the Paths of Light and of Planets by the Coefficients of a Characteristic Function which generalized and extended this optically devel oped theory into mechanics, and extended his treatment from two to three bodies. Other works on this topic were his paper in 1834 On a General Method in Dynamics, and an essay in 1835 He demonstrated that his method when applied to the then known solar system of ten planets, simplified the problem of solving the sixty differential equations of Lagrange, to the search for a single function which satisfies two partial differential equations of the first order and the second degree. Hamilton proposed a general treatment for an attracting system of bodies by his reduction of the mathematics "to the study of one central function". His method was as in his ray theory to reduce the problem to one based on the initial and final co-ordinates of the body, which resulted in the characteristic function $V$ being a function of the $6 n$ co-ordinates of initial and $f i n a l$ positions and the Hamiltonian H. This energy operator was constant along any real path, but would vary if the initial and final points were varied.

In the first essay he considered methods of approximating the characteristic function as applied to planets and comets and introduced a new auxiliary function called the principal function $S$. In the second essay he introduced his famous canonical equation of motion and deduced that $S$ was equal to the time integral of the Lagrangian between fixed points. The statement that the variation of this integral must be equal to zero is now referred to as Hamilton's principle.

Hamilton focused on the fundamentals of algebra in a paper Algebra of Pure Time that once again had at its heart "the great idea" of his calculus of variation. In this study 
he attempted to place the algebraic notions of negative and imaginary numbers on a firm foundation which was for him to be found in "the ordinal character of numbers". He believed these must be ordered on an intuitive basis in time and that this ordering was more deep-seated in the human psyche than the intuition of order in space.

Hamilton was philosophically determined to replace the Cartesian Algebra of the Point by one based on the intersection of two lines. Four elements were necessary to determine the relation of one line in space to the other:

(i) The relation which the length of the line bears to the length of the other line;

(ii) The angle through which one line must be turned to coincide with the direction of the other;

(iii) The plane in which the two lines lie;

(iv) The determination of this flane with respect to some reference plane.

The combination of the four elements then forms the Calculus of Quaternions. He developed this theory, appropriately one might say, in stages; firstly he dealt with Couples, then Triplets and finally Quaternions. The value of the couple $(a, b)$ depends on the order as well as the manitude of its constituent step and in this study he identified the operator $i$ to change a real number a, intc an imaginary number $\checkmark \overline{-1} a$ by rotating this on the Argand Diagram by Gjo, and so on, where $i^{2}=-1$. He concluded that $i$ was equivalent to $\sqrt{-1}$ and that this was "a perfectly real operation". Given this result he moved on to consider the triplet (a,b c) and he was motivated by a desire to connect in some new way "calculation with geometry.

The "triple algebra", so called by de Morgan, led directly to the Quaternions, since Hamilton discovered that these oper- 
ators were non-commutative, and it was his genius to grasip that it was possible to develop a meaningful and consistent algebra which is not commutative, the first in the history of mathematics. The date of inspiration is indelibly recorded in the annals of history by Hamilton writing his famous equation on Brougham Bridge on the Royal Canal on the 16 th October 1843. Boole subsequently demonstrated (based on Hamilton's suggestion) that there is not one algebra but many with a wide range of fundamental postulates [9].

Hamilton introduced the dot product and vector product in his algebra which contained within the one algebra a total description of three-dimensional space. He introduced the two well known operators in modern mathematical physics

$$
\Delta=i \frac{d}{d x}+j \frac{d}{d y}+k \frac{d}{d z}
$$

and

$$
\Delta^{2}=\left(\frac{d}{d x}\right)^{2}+\left(\frac{d}{d y}\right)^{2}+\left(\frac{d}{d z}\right)^{2}
$$

the latter named 'del' subsequently by Gibbs. This work led directly through the mediation of FitzGerald to the theory of Relativity developed by Einstein who essentially extended this theory to four dimensions.

The importance of Hamilton's work for these later developments can be judged from a large number of mathematical papers on related topics, such as his 1861 memoir On Geometrical Nets in Space. It has been pointed out that pauli's spin matrices introduced in 1927 are simply Hamilton's i,j,k [10] and that Hamilton first made the discovery of the distinction between group and wave velocities.

His diverse contributions have been recognised by a number of significant scholars. The work of Hankins [5] has however provided the most detailed overview of his life; while that of his contemporary Graves [11] gives the best appreciation of his standing in his own day. His honours were rather limited, and although he was President of the Royal Irish 
Academy from 1837-1845, won the Royal Society's Medal in 1836, and had the enviable reputation in his own day of being the leading man of science in Ireland, he never was elected a Fellow of the Royal Society.

\section{THE DYNAMICS DF COMPETITION}

The mathematical competition between MacCullagh and Hamilton was an essential component in propelling these men to the greatness they both achieved. The personal rivalry between the two men has been well documented [3] and was produced principally by MacCullagh's jealous reactions to a series of discoveries by Hamilton. Both men were attacking the same problems by different methods and perhaps it was not therefore surprising that MacCullagh should feel that his studies had already anticipated some of Hamilton's discoveries, most notably conical refraction. While this rivalry became very bitter and personal it was a conflict engendered by the international controversy over the nature of light and the importance given at the time to unification of the sciences of mechanics and 1 ight $[12]$.

In this age of great international competition mathematics was the acknowledged battle centre for the sciences and the ultimate theory of the mechanical philosophy. For Maccullagh this golden grail was to be won by providing a geometrical solution to the problem based on a mechanical model of the ether, the long sought Cartesian synthesis. For Hamilton on the other hand a new algebra was seen as the solution and his theoretical foundation of the physical sciences would have required no mechanical model or ether modelling.

The conflict in the two men's methodologies was between that of the applied mathematician and the pure mathematician, the materialist and the idealist, and the Newtonian and continental schools. 
Maccullagh was in the last analysis a disciple of Bartholomew Lloyd and a natural philosopher in the Newtonian sense of the word, while Hamilton the self-taught mathematician was unequivocally a supporter of French mathematical "physique". This difference can be seen in their views on the central question under debate internationally at the time, the wave-corpus cular controversy [13] over the nature of light. Maccullagh was a "faint hearted supporter" of the wave theory with Newtonian doubts that the wave theory's physical basis had not been rigorously established. Hamilton was a true "supporter" of the wave theory [14]. Dublin also boasted in its midst at this time Dionysius Lardner, a collaborator of the Edinburgh Newtonians who formed the central core of the "abjectors" to the wave theory [15].

Dublin in the 1830 s was opened up to the maelstrom of this international research controversy and this provided the dynamic for what were probably the most significant developments in the history of Irish mathematics. The fact that Dublin mathematicians were able to move to the forefront of science at this time was because Bartholomew Lloyd [16] had reformed the curriculum of Dublin University and made it possible for the young lions of the emerging school to base their research on the most advanced paradigms of the day. However, from this period on, the research traditions in Dublin mathematics were based on its own bifocated traditions of geometry and algebra and not merely on foreign inspiration.

\section{REFERENCES}

1. McDOWELL, R.B. and WEBB, D.A.

'Trinity College Dublin 1892-1952' (Cambridge, 1982), p. 47 .

2. "Obituary MacCullagh", R.I.A. Proc., Vol.4 (1847-1850) pp. 103-117. 
3. O'HARA, J.G.

'Humphrey L1oyd', (1800-1881) and 'The Dublin School of the Nineteenth Century', (UMIST, 1979).

4. JELLETT, J. and HAUGHTON, S.

'Collected Works of MacCullagh', (Dublin, 1880) includes MacCullagh's The Dynamical Theory of Light.

5. HANKINS, T.L.

'Sir William Rowan Hamilton', (Baltimore: John Hopkins U.P., 1980).

5a. O'DONNELL, S.

'William Rowan Hamilton: Portrait of a Prodigy', (Boole Press, 1984).

6. HAMILTON, W.R.

'On Caustics', submitted to Royal Irish Academy on December $13 \mathrm{th}, 1824$.

7. LLOYD, H.

Trans. Roy. Irish Acad., Vol. xuil, p. 145 (1833).

8. SYNGE, J.L.

"Hamilton's Method in Geometrical Optics", $J$. of the optical Soc. of America, Vol. 27, pp. 75-82 (1937).

9. MacHALE, D.

'George Boole; His Life and Work', (Boole Press, Dublin, $1985)$.

10. WHITTAKER, E.T.

Mathematical Gazette, Vol. 25 (1941) 300.

11. GRAVES, R.P.

'Life of Sir William Rowan Hamilton', (Dublin: Hodges and Figgis, 1882-1891).

12. The development of specialization at this time in the physical sciences was intimately related to the mathematization of these sciences. See for example Crosland $M$. and Smith, C., 'The Transmission of Physics from France to Britain (1800-1840): Historical Studies in the Physical Sciences', (John Hopkins, 1978). 
13. For a contemporary view on this debate with a special reference to Dublin, see Lloyd, H., 'Report on Optics' British Association for the Advancement of Science, (Dublin, 1835).

14. The views of MacCullagh and Hamilton on this question were revealed in the debate at the 1843 British Association meeting at Cork.

15. Lardner was appointed the first professor at the new Londan University College in 1827 by Lord Brougham, the leading Newtonian who was based in Edinburgh and associated there with David Brewster, the other leading supporter of the corpuscular theory.

16. The reform of the curriculum in Dublin University was dealt with in Part 1 of this article.

lichoele of scicince,

liegianat Technical reflege,

C.1 $2 \ell$ ow. 